\title{
Understanding the Role of General Dentists in the Process of Screening and Early Diagnosis of Oral Premalignant And Malignant Lesions - Brief Literature Review
}

\author{
Jordan Gjurcheski ${ }^{1}$, Danica Popovic-Monevska² \\ ${ }^{1}$ University Dental Clinical Center "Prof. Dr. Bojo Andreski", European University, Faculty of Dentistry, Skopje, Republic of Macedonia; ${ }^{2}$ Clinic \\ for Maxillofacial Surgery, Dental Faculty, Ss. Cyril and Methodius University Skopje, Republic of Macedonia
}

\begin{abstract}
Citation: Gjurcheski J, Popovic-Monevska D. Understanding the Role of General Dentists in the Process of Screening and Early Diagnosis of Oral Premalignant And Malignant Lesions - Brie Literature Review. Maced J Med Sci. 2012 Dec Literature Review. Maced J Med Sci. 2012 Dec $\mathrm{g} / 10.3889$ MJMS.1857-5773.2012.0250.

Key words: oral premaliglant lesion; screening for oral cancer; oral cancer; general dentist; oral precancerous lesion; early diagnosis; toluidine staining; autofluorescence.

Correspondence: Jordan Gjurcheski. University Dental Clinical Center "Prof. Dr. Bojo Andreski", European University, Faculty of Dentistry, Kliment Ohridski bb, 1000 Skopje, Republic of Macedonia. E-mail: jordan.gjurceski@eurm.edu.mk

Received: 25-Apr-2012; Revised: 10-Jun-2012; Accepted: 19-Jul-2012; Online first: 07-Nov-2012

Copyright: @ 2012 Gjurcheski J. This is an openaccess article distributed under the terms of the Creative Commons Attribution License, which permits unrestricted use, distribution, and reproduction in any medium, provided the original author and source are credited.

Competing Interests: The author have declared that no competing interests exist.
\end{abstract}

\section{Abstract}

Aim: To indicate about the need for involving general dentists in the process of screening and early detection of malignant and premalignant lesions. To make brief literature review, which lesions are included under the term "oral premalignant lesions", and which lesions should be excluded from this nomenclature, according to the contemporary views and conceptions. To gather in one place the latest information about new diagnostic procedures used worldwide for screening and early diagnosis of premalignant and malignant lesions of oral mucosa.

Material and Method: Searching was conducted by electronic selection of published papers over the largest database of published papers, PubMed (http://www.ncbi.nlm.nih.gov/pubmed/), and its subsequent ramifications PubMed Central (http://www. ncbi.nlm.nih.gov / pmc /), as well as through the database of Google Scholar (http://scholar.google.com/).

Discussion: Of particular importance it is the need to highlight the great role of the general dentist in the process of screening and early detection of oral cancer. Also it must be emphasized the role of the patient, his education and socio-economic status.

Conclusion: There is need for additional education of dentists about contemporary understanding of this disease. Also there is a need for education of the population about the oral cancer.

\section{Introduction}

Early diagnosis of certain diseases is crucial fact that determinates the success of therapy. That is the case with oral cancer too. Unfortunately, one of the most important reason for high mortality and poor survival rate after treatment of oral cancer (despite its high malignancy) is precisely the absence of its early diagnosis.

Certain conditions of the oral mucosa are known to have greater potential for malignant transformation than the surrounding oral mucosa. These clinical changes are known as oral premalignant lesions (precancerous conditions). However, to observe these changes at regular dental examination with white light, you must make a detailed examination of the oral mucosa. Very often, initial premalignant and malignant changes in oral mucosa are not visible to the diagnostician and do not have any particular and specific clinical symptoms. 
Day by day there is an improvement in the diagnostic process and new methods and tests for screening and early detection are presented. This process is followed by more simple way of usage in order to allow the general dentists to use these methods. The main goal of this improvement is to keep the level for early detection of premalignant and malignant lesions to the facilities of primary and secondary health care (general dental offices and specialist office for oral surgery and oral pathology), and to stop the everyday tendency for transferring this process to the facilities of tertiary health care (Department of Maxillofacial Surgery).

Of all oral malignancies originating from the oral mucosa, 85 to $95 \%$ belong to the group of squamous cell carcinoma, while the rest of them belong to sarcomas, melanomas, tumors of the small salivary glands, lymphoma, and metastatic deposits of malignant tumors of different localization in the organism [1, 2]. Very often, the term "oral cancer" in dental literature is used to identify the pathological diagnosis - squamous cell carcinoma.

Oral cancer should never be forgotten when discussing the epidemiology of malignant tumors worldwide. Although its incidence is estimated from 3 to $4 \%$ of all malignant tumors, the degree of survival is on a very low position. Another disappointing fact is that in the past 50 years that position is not significantly altered because of late diagnosis of this disease [3]. Annually 28 000 [3] to 35,000 [4] new cases with oral cancer are diagnosed in the United States. About 8000 [4], or 9000 [3] deaths annually are caused precisely as a consequence of oral cancer. Worldwide, 400,000 new cases per year are diagnosed with this disease [5]. According to these numbers, oral cancer is shown as more deadly neoplasm than breast cancer, cancer of the cervix and prostate neoplasm. That's why it was established that oral cancer kills one man every hour, every day [6, 7]. It is paradoxically what Kevin states that oral cancer is one of the cancers that are treated with the greatest success, but if detected in its early stage. He argues that with appropriate early intervention, five-year survival rate of these patients is even more than 90\% [4]. According to Gillenwater oral cancer is among the top 10 most common neoplasms worldwide [8]. Her statement coincides with the opinion of other authors [3-7] regarding the degree of survival. The prevailing opinion that five year survival rate of these patients many years has not changed and ranges from $50-55 \%[2,8]$. According to Neville, the localization of oral cancer plays an important role in the survival rate of these patients [2]. He states that five-year survival in patients of white race in the U.S. is $47 \%$ in carcinoma of the tongue, $33 \%$ in carcinoma of floor of mouth, and $95 \%$ of oral cancer of the lip. Fiveyear survival was $75 \%$ for those patients with localized changes, while only $16 \%$ for patients with advanced stages of disease, because in most of the cases it has been diagnosed when the cancer is in stage 3 and 4 , already with present metastases in the lymph nodes [3, $9,10]$. According to Lingen, at the first examination $60 \%$ of cases appeared with oral cancer at stage 3 and 4 [5].

Epidemiological data of premalignant lesions are difficult to obtain. Perhaps the reason is precisely the non-uniformity of opinions in the dental community about "what exactly is a premalignant lesion" and "which of suspicious lesions can be placed under the umbrella of the term premalignant lesion". Another reason is the inconsistency and unreliability of data, in other words, nonexistence of the proper way for collecting and processing the data from the network of dental offices.

Epidemiological data on oral cancer and premalignant lesions for the territory of Republic of Macedonia, is not known.

Five-year survival rate after treatment of oral cancer and premalignant lesions, is inversely proportional to the stage in which these lesions are detected. It is a bit frustrating fact that oral cancer is presented on the best available place on the human body, oral mucosa, a field that is most accessible for visual and tactile examination, yet still holds the high place on the scale for late detection and poor post-treatment prognoses, compared with other neoplasms in human organism.

As we can conclude, the key for moving this disease from its unchanged position on the ladder of death lies in the hands of the population of general dentists in one society. Lack of preventive measures and measures for early detection of oral cancer by dentists is not only a problem in our country but it is worldwide problem as well. Many dentists claim that they perform preventive examinations for early detection of premalignant and malignant lesions, but some studies indicate that that's not true, and that there is a lack of knowledge of the problem of etiology and diagnosis of this disease $[3,11]$.

Considering and comparing the literature data related to the issue of oral cancer, a necessity for writing this review paper was born, as a base for larger literature and epidemiological research about the state of oral cancer in the Republic of Macedonia in terms of its possible etiologic factors, the success of therapeutic 
procedures, survival rate and, above all, contemporary opportunities for early diagnosis of malignant and premalignant lesions of oral mucosa. Motivated by this need, we set and specify the aims of this paper. Before setting the aims of our article, we would like to highlight the fact that this article will not give clear guidelines and protocols for screening and early detection of oral premalignant and malignant diseases, but it will make the first little step as a stimulus for solving this current issue. Our first aim is to raise the awareness of general dentists from one society about their role in the process of screening and early detection of malignant and premalignant lesions. Secondly, to make brief literature review, and give clear picture to the general dentists, which lesions are included under the term "oral premalignant lesions ", and which lesions should be excluded from this nomenclature, according to the contemporary views and conceptions. Lastly, to gather in one place the latest information about new diagnostic procedures that are used or are still under examination worldwide for screening and early diagnosis of premalignant and malignant lesions of oral mucosa (Table 1).

Table 1: Aims of the review paper.

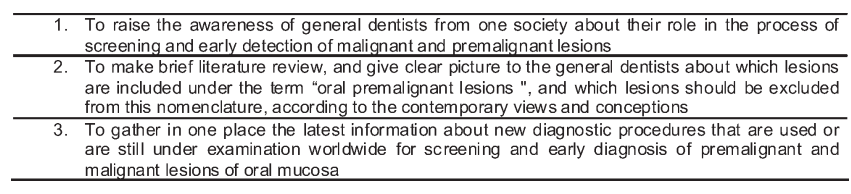

\section{Material and Method}

To achieve the aims of this paper, it was necessary to perform research through the published literature all over the world. Research was made through already established and published textbooks of Oral and maxillofacial surgery and Oral pathology that are used in dental schools and through the latest articles published in indexed dental and medical journals. There were no date limitations for the researched literature, because we thought that data from older articles would be also useful to compare, especially in the process of evaluating the survival rate of these diseases. We searched mostly English literature, but also Macedonian, Serbian and Croatian literature data were taken into consideration. Searching was conducted by electronic selection of published papers over the largest database of published papers, PubMed (http://www.ncbi.nlm.nih.gov/pubmed), and its subsequent ramifications PubMed Central (http:/ /www. ncbi.nlm.nih.gov/pmc/), web page for free on-line editions of magazines and papers. Also, part of the literature data was received through the database of Google Scholar (http://scholar.google.com/). For searching in these databases were used following keywords: premalignant lesions, oral premalignant lesions, precancerous lesions, early diagnosis, oral cancer, oral carcinoma, oral cancer screening, early detection of oral cancer, general dentist and oral cancer, autofluorescence, Velscope, brush biopsy, brush cytology, toluidine staining.

Obtained papers were read, compared, and the most important parts were transferred in the text of this paper.

\section{Discussion}

Comparing the reviewed data from the literature, discussing with relevant experts in the maxillofacial surgery branch, and also considering the situation with oral cancer in the Republic of Macedonia, we can say that the situation is similar all over the world. It seems that the more advanced way of tertiary health care is introduced, or the more developed and improved therapeutic modalities for oral cancer are used, the less attention is paid on emphasizing and popularizing of screening methods and techniques for early diagnosis of premalignant and malignant lesions of the oral mucosa. Still as a consequence of that, large number of treated patients end with fatal outcome after frequent recurrence, and metastatic changes of this disease. Our opinion is that the problem for the unchanged situation in this field lies on several levels: 1. Lack of enthusiasm and underestimating the role of primary care (general dentists) in early detection of disease 2 . Insufficient knowledge of the problem with oral premalignant lesions and early malignant lesions and 3 . Insufficient processing and presenting of new diagnostic methods for early detection of premalignant and malignant lesions of oral mucosa, and their slow penetration into the primary and secondary dental care.

\section{Role of primary and secondary care system in the process of screening and early detection of oral premalignant and malignant lesions}

As we mentioned previously, oral cancer occurs in one of the most accessible places in the human organism. The oral mucosa is a medium that is available for both visual and the tactile inspection. Therefore, quite paradoxical is the fact that until now, not much is done in the field of screening and early detection of this disease. 
In recent medical past there are a lot of examples that show how the introduction and promotion of screening programs have a positive influence on the prognosis of some malignancies with high mortality (Pap test for early detection of cervical cancer, and mammography as a screening tool for breast cancer [3, 5, 12]). This year with a decision of the Government of the Republic of Macedonia, a Program for early detection of malignant diseases in Republic of Macedonia was prepared, which approves screening methods for three types of cancer: cervical cancer, breast cancer and colorectal cancer [13].

Since these screening programs and methods have their cost, and certainly the possibility of error (false positives) their use must be justified and cost effective. Therefore, strict criteria are needed for when and where is reasonable to perform these screening programs on population masses [5]. According to the National Committee for screening in the UK, it is necessary to satisfy a list of 22 criteria before initiating a screening program [14]. For oral cancer, it is sufficient to meet at least 3 of these criteria to justify the existence of a screening program. Some of these criteria are given in Table 2 [15].

The role of general dentist as well as specialists from relevant dental branches, in the process of screening and early detection, is undoubtedly great. If there would be a national program for screening and early detection of oral cancer, dental offices (whether general practice or specialists) all over the state, would be the tool through which this program would be performed.

Even though the basic and standard oral examination for early detection of oral cancer takes only 90 seconds, compound of visually and tactile examination, a very few dentists perform it [16] (However, this refers to an ordinary everyday oral examination for detecting any oral changes. For detail oral investigation, there is need for longer examination).

Much of the dentists claim that they conducted this examination for oral cancer among their patients,

Table 2: Criteria for the implementation of a screening programme according to UK National Screening Committee [15].

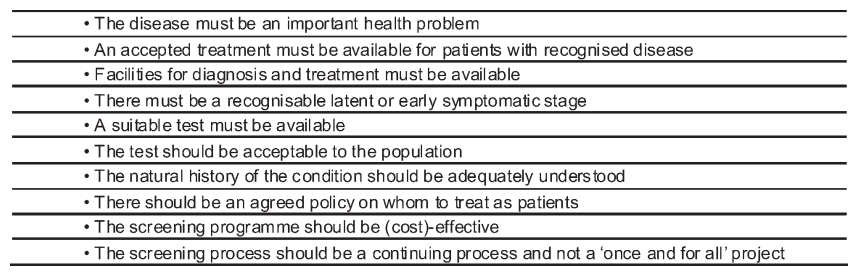

but studies show that among the broad mass of the dentists there is lack of knowledge regarding the field of oral carcinogenesis and diagnostics [11]. A number of surveys conducted among dental population suggest that there is a desperate need for additional education and update of already-known facts regarding the prevention of oral cancer and its early detection [16]. Horowitz et al. after the study conducted in 2000 among dentists across the United States, concluded that they require systematic training that will emphasize the need and importance of: 1 . Collecting a full medical history of patients, including history of any risk factors such as use of tobacco and cigarettes, and 2 . Increasing the frequency of examinations of the oral mucosa [3].

American cancer society recommends screening for cancers of the head and neck, including oral cancer every three years in asymptomatic patients between the age of 20-40 years, and annually in asymptomatic patients aged over 40 years. Smokers and alcohol abusers should be examined annually regardless of their age [17].

It seems that dentists are not aware of their power in this field, about the fact that 90 seconds of their time, in which they'll make detail examination of the oral mucosa, can raise them on a pedestal as doctors heroes, and that with minimal effort, they can make a lot for the patient and for the dental profession, if they detect the disease in its early stages.

We should put an accent on another important factor in this circle. Patients have very important role for early diagnosis of oral premalignant and malignant lesions too. The level of education and socio-economic status of patients, especially in developing countries are in indissoluble connection with the dentist's desire to promote the process of screening and early detection of oral cancer. One of the main problems is unconscious and lack of knowledge in patients about "what is oral cancer." Unfortunately, oral cancer gives its first noticeable symptoms when it is already in an advanced stage. Patients, especially those with high risk (smokers and alcohol consumers) should know that their frequent visits to the dentist for preventive purposes may be of crucial meaning for their life. Educating and raising awareness among patients about this disease would be of great help for dentists.

\section{A few words about the contemporary understanding of oral premalignant lesions}


According to some authors the term "premalignant disease" includes localized lesions, as well as generalized conditions, and therefore we can speak about premalignant condition, and premalignant lesions [ ${ }^{20}$. According to them, a premalignant condition is a generalized disease or condition which may not give any changes in oral mucosa, but may be associated with greater risk of developing oral cancer. The term "premalignant lesion" refers to a morphologically altered tissue that is more likely to develop malignant transformation, compared to normal mucosa $]^{23}$. Most premalignant lesions are clinical conditions, not pathologically definite lesions, and it is very difficult to differentiate them from other oral changes, with benign nature. Oral premalignant lesions are diagnosed by exclusion, they don't have their own characteristic pathohistology features, and therefore their stigmatization as oral premalignant lesions must be very carefully done, after you exclude all other possible benign conditions of the oral mucosa. Since many oral changes were considered as premalignant lesions in the past, after a lot of studies and investigations, the broad umbrella of the term "oral premalignant lesions" narrows day by day. A lot of oral changes that have completely different etiologies, other than carcinogenesis of oral cancer, and those lesions that are rarely shown to have malignant transformation, are removed from this classification. Most authors and papers suggest that premalignant and early malignant lesions are clinically manifested as leukoplakia or erithroplakia, and if properly diagnosed, they are most common, and probably the only real oral premalignant lesions $[1,2,8]$. In the following text, very briefly, we will mention those lesions and changes that are placed under the roof of the term "oral premalignant lesions", in contemporary literature.

Leukoplakia. Leukoplakia is a clinical term that was first used in 1877 by Shwimmer, to describe white lesion on the tongue that probably was representing syphilitic glossitis. From then until today, there is a major change in the opinion about what is leukoplakia. Many lesions are excluded from this classification. According to the World Health Organization (WHO) leukoplakia is "a white patch or plaque that cannot be characterized clinically nor pathologically as any other disease" [18]. There must be emphasized that the term leukoplakia may be used only as a clinical diagnosis, because it is not related to any specific pathohistology features, and should never be used as a definitive microscopic diagnosis. Generally, leukoplakia is an asymptomatic lesion and clinically presents as white or creamy lesion that may be flat, slightly raised, with a convoluted surface, or smooth. Its important clinical feature is that it cannot be removed from the surface with any mechanical noninvasive procedure. If a white change, found on the oral mucosa may be diagnosed as another condition (lichen planus, candidiasis, leokoedema etc.) then that lesion should not be taken into account as leukoplakia $[1,2]$. Day by day the circle of this clinical diagnosis is narrowing and more a lot of oral lesions are thrown out of that circle. For example, often, toothless areas of the alveolar ridge that are not properly prostheticaly treated, show thickened hyperkeratotic changes that resemble leukoplakia. Some histological studies have shown that it is just a simple defensive hyperkeratotic response to chronic irritation, and extremely rare there is dysplastic transformation of these cells. That's why many experts classify these changes into a separate diagnosis "frictional keratosis". Also, secondary hyperkeratotic changes that occur as a result of chronic cheek biting (morsicatio buccarum) or tongue biting (morsicatio linguarum), should not be placed under the umbrella of leukoplakia, because these changes are reversible. Also, nicotinic stomatitis and nicotine keratosis should be excluded from the shelter of this clinical term, because changes have well known etiology and are defined as separate diagnoses. As the circle of lesions that are named as leukoplakia narrows, there are more trials that suggest more frequent malignant transformation of leukoplakia. According to certain authors there is broad percentage area of malignant transformation of this change, varying from $0,13-17,5 \%[10,19-21]$.

Erithroplakia. Similar as leukoplakia, erithroplakia is clinical term that was primarily used to describe a pathologic lesion quite different from today's opinion about what is eritrhoplakia, so over the years, the diagnostic circle narrows, so the clinical diagnosis is also diagnosis of exclusion. By definition erithroplakia is red patch or plaque that can, neither clinical nor pathologically, be attributed under any other diagnosis of the oral mucosa [18]. Clinical often appears as a painless change, but some patients complain of burning of the mucosa. It appears as red stain or plaque area that has velvety texture. Although erithroplakia is not as common diagnosis as leukoplakia, pathologically this reddish lesion shows more signs of dysplasia than leukoplakia. Shafer and Waldron made parallel investigations on leukoplakic and erithroplakic samples and found that of 65 examined cases with erithroplakia, all showed signs of varying degrees of dysplasia (51\% - squamous cell invasive carcinoma, 40\% - Carcinoma in situ and 9\% - mild to moderate dysplasia) [22]. Thus, properly diagnosed true erithroplakia, is much more 
worrying lesion than leukoplakia.

Here it should be emphasized that very often these changes can occur as a mixed form, also known as erithroleukoplakia (speckled leukoplakia or speckled erithroplakia) [1, 2].

Oral submucous fibrosis. Oral submucous fibrosis represents a premalignant condition that can be seen in India and Southeast Asia. It is a chronic mucosal disorder that is associated with the habit of chewing betel quids. This change is characterized by mucosal rigidity that leads to the appearance of trismus, pain when eating spicy foods and difficult speaking and swallowing. Unlike mucosal changes caused by nicotine (nicotine stomatitis and nicotinic keratosis) this change is not reversible after discontinuation of the bad habit. Longitudinal studies have shown a degree of malignant transformation of this lesion in $7.6 \%$ of cases [23].

\section{Methods and tests for screening and early diagnosis of oral premalignant and malignant lesions}

In order to promote oral health, improve the process of early detection of oral cancer and increase the efficacy of therapy, by increasing the percentage of five-year survival rate, dental industry, supported by scientific circles, often present new devices, apparatus and methods on the market for screening and early detection of this disease. From this broad ocean of tests and methods, based on clinical and molecular level, some of them are used for a long time, and have documented numerous scientific trials, but some of them are still unexplored, waiting to be scientifically validated and to start their mass-usage.

As gold standard for diagnosing oral cancer still remains tissue biopsy with pathological confirmation of the changes. But performing this accurate and reliable diagnostic procedure requires specially trained staff, and it is not economically and morally viable for mass use. Therefore there is constantly need of researches for appropriate methods for mass usage. Each test or screening method for early diagnosis will be compared with the gold standard, and thus determined its sensitivity and specificity. In this part of our review paper briefly we will mention all the methods and tests for early diagnosis that we found in the researched. Collecting and analyzing these data, we tried to divide them by way of use. The classification is presented in Table 3.

Staining with tolonium chloride (toluidine blue). Tolonium chloride is a metachromatic dye that
Tàble 3: Methods and tests for screening and early diagnosis of oral malignant and premalignant lesions.

\begin{tabular}{|c|c|c|}
\hline Methods for vital staining & Cytology methods & Direct visualization of tissue \\
\hline $\begin{array}{l}\text { - } \begin{array}{l}\text { Tolonium chloride } \\
\text { (toluid ine blue) }\end{array} \\
\text { - Hematoporfyrin }\end{array}$ & $\begin{array}{l}\text { Brush biopsy } \\
\text { Pathohistology } \\
\text { analysis } \\
\text { Computer } \\
\text { assisted analysis } \\
\text { (Oral CDx) }\end{array}$ & $\begin{array}{l}\text { - } \begin{array}{l}\text { Chemiluminescence } \\
\text { (ViziLite) } \\
\text { - Autofluorescence } \\
\text { (VELscope) } \\
\text { - Usage of } \\
\text { photosensitizing and } \\
\text { molecularly labelet } \\
\text { contrast agents } \\
\text { - Optical and diffuse } \\
\text { spectroscopy } \\
\text { - Confocal microscopy } \\
\text { - Optical coherent } \\
\text { tomography }\end{array}\end{array}$ \\
\hline
\end{tabular}

colors malignant cells and make them clinically visible. That is not a case with the cells from normal mucosa. It seems that this is the oldest method for this purpose, because it is known that it has been used for many decades in order to delineate the malignant mucosa of the cervix and in the oral cavity. There are couple of assumptions about the way this method works. Depending to one of them, tolonium chloride is been ingested by the nuclei of malignant cells, thus manifesting the increase synthesis of DNA [8]. Depending to other theory, it is meant that toluidine freely passes between irregularly arranged malignant cells [3].

The procedure is performed so that the patient rinses the mouth with this dye, after which the physician examines the oral mucosa looking for blue colored fields. Malignant lesions are stained dark blue, while dysplastic lesions are stained with different shades of blue depending on the degree of dysplasia [24]. It is possible a healthy mucosa to absorb the color, but after rinsing with acetic acid, these fields has been washed. Normally, keratinized and uneven surfaces retain some of the color (dorsal surface of the tongue, gingival sulcus). According to Rosenberg this method has a sensitivity of $93.5 \%$ to $97.8 \%$ while specificity that varies from $73 \%$ to $92,9 \%$ [25]. From their observations and comparisons of literature data, Gillenwater et al. conclude that staining with toluidin blue may be useful as a prognostic marker for malignant potential of oral premalignant lesions in high risk patients [8].

\section{Staining with hematoporphyrin.} Hematoporphyrin derivatives, similar to the tolonium chloride are more retained in abnormal compared to normal epithelial cells. But, because this staining is less visible on white light (from dental reflector) some authors recommend this method to be used in combination with blue excitation light (380-450 nm). Using this light, the normal mucosa is shown as colored blue, while lesions that retained color, are displayed as red fields [26].

OralCDx - computer assisted analysis of samples taken by brush biopsy. This is a new method 
in which cytology samples taken with a special brush as in Pap test, are analyzed with a special program designed for that purpose. This method was created in order to evaluate those changes with high clinical susceptibility for malignancy. To avoid pain and discomfort in patients, first this test is performed, so if you get a "positive" or "abnormal" result, patient must be traced to the gold standard, tissue biopsy, and pathological reevaluate the case [3, 8].

Saliva as a diagnostic tool. Saliva as a medium is a place where there are a number of incidentally peeled cells. Therefore, it is been increasingly questioned and quite attractive for researchers to take advantage of cytological and molecular analysis. Some new research shows that there is a difference in DNA and RNA expression in cells found in saliva in patients with oral cancer, and patients with normal mucosa [27]. However, this is a broad field of research, and offers enough options, and therefore, has not been sufficiently clarified and explored. After a period of time, if this method is properly examined and verified, it can climb high on the scale of these diagnostic tests. Until then, it can be used only as an information about its existence, and as a possible field for prospective researches.

Chemiluminescense - ViziLite. This method is a noninvasive procedure that detects surface suspicious lesions. Patient rinses the mouth with $1 \%$ acetic acid, and then in a dark room under blue light (short wavelength: 430, 540 and $580 \mathrm{~nm}$ ) the clinician examines the oral mucosa. Normal mucosa is presented as a dark zone, while suspicious lesions reflect light and are seen as white areas. Presumably because of dense chromatin material and density of nuclei, abnormal cells reflect light, while normal cells absorb it and therefore appear as dark areas [28]. Most tests are based on subjective criteria such as brightness, sharpness and texture, and therefore it is not surprising that they get contradictory results.

Autofluorescence - VELscope. The ability of normal tissue for autofluorescence, was used many years before to detect premalignant and malignant lesions of many organs (lungs, cervical mucosa, skin, etc.), and recently this method was actualized as a method for screening and early detection of lesions of oral mucosa. The healthy tissue at the molecular level contains substances called fluorophores such as NADH (nicotine amide adenine dinucleotide), FAD (flavin adenine dinucleotide) and cross-links of collagen and elastin, which when they are excited with light rays with a specific wavelength, have the ability to produce its own fluorescence-autofluorescence [8]. Unlike healthy cells and tissues, in abnormal - cancer cells, there is a change in structure (hyperkeratosis, hyperchromatism, and increased nuclear and cellular pleomorfism), change in metabolism (the concentration of FAD and NADH) of epithelial cells, and changes in subepithelial stroma. These changes directly change the distribution of fluorophores, so there are changes in their interaction with blue excitation light [3].

To use this property on different tissues, dental industry provided different kind of apparatus, specially designed for different anatomical regions. VELscope is a kind of lamp that produces intense blue excitation light with frequency from 400 to $460 \mathrm{~nm}$. Oral mucosa is observed under a special lens (from the set), so normal epithelium is detected as green avtofluorescent surface, while the abnormal parts are seen as dark areas. A number of authors have examined the effectiveness of this method and most of them confirm the sensitivity above $90 \%$ and specificity above $80 \%$ for this device [29, 30].

Latest trials and results give hope that using this method will justify its existence and it will be presented as a method for mass screening.

Photosensitizing agents. Knowing the possibility of autofluorescence of tissues can be seen with special devices, dental science went step further, and concluded that visualization would be far better if we help this process by special substances called photosensitizers. It was observed that after using photosensitizing agents locally or systemically, the abnormal tissue has the capability of fluorescence. Optical active contrast agents can be intensely red fluorescent dyes, porphyrin derivatives and nanoparticles. It is known that in abnormal malignant tissue, there is an increased concentration of EGFR (Epithelial growth factor). Because of his hyperexpresion, it represents an appropriate molecular target that can be detected and can indicate a possible malignancy. Photosensitizing agents bind with monoclonal antibodies directed towards EPGF, and so once they bind to the tissue, they can be visualized. Soukos et al. used fluorescent dye indocianin Cy 5.5, Hsu et al. used intensively red fluorescent dye (Alexa Fluor 660), whereas Sokolov et al. used gold nanoparticles, all these, conjugated with monoclonal antibodies against EGFR [30-33]. There is a need for prospective trials for this procedure before it gets into routine screening methods.

Optical and diffuse spectroscopy. The 
difference between these two methods is the size of examined surface. A fiberoptic probe is used for optical spectroscopy in order to examined small areas, while diffuse spectroscopy is used for examination of larger areas. The essence of this method lies in the ability to measure the emission and absorption of different wavelengths of visible and invisible light. Both methods are based on the same principle. Registered optical spectrum of the tissue provides information about biochemical composition and structure of the tissue. Altered biochemical composition and structure of tissue that indicate malignancy, disrupts the normal absorption, fluorescense and ability to scatter the rays through the tissue. Information on these changes is obtained in the form of graphs and require additional education to read them. Raman spectroscopy represents a kind of diffuse spectroscopy, about which nowadays a lot of trials are done $[3,26,34]$.

Confocal microscopy. A modern method used for microscopic investigation of tissue in vivo but without making pathologically samples. Using different techniques of focusing and reflection of light, the technique manages to record a variety of tissue contrasts, and thus to visually separate the nucleus from the cytoplasm of the cells. Change in the nucleus / cytoplasm ratio can be used to differentiate healthy from malignant tissue. Because of the fact that this method has very limited capacity for penetration $(250-500 \mu \mathrm{m})$ and because of its cost and expensive maintenance, there is little interest for mass usage of this method $[3,18]$.

Optical Coherence Tomography. Until now this is very successful method used to diagnose retinal pathology. In the past years it already takes his place in oral pathology. It works similar to ultrasound, except the fact that is uses infrared beams to obtain high resolution images of tissue. Since it has power of penetration up to $2 \mathrm{~mm}$, it is suitable for examination of lesions of oral mucosa $[3,8]$.

\section{Conclusions}

As we can see from the current text there is broad ocean of opportunities for screening and early detection of oral premalignant and malignant lesions. Some of them are well-researched and already established and approved, and some of them are still in experimental stage, waiting to find their place in the population of dentists. All data received from reading, discussing and comparing the literature, led us to several conclusions:
1. The role of general dentists and specialists in certain dental branches is undoubtedly the key for advancing and actualizing the problem with oral screening and early diagnosis of premalignant and malignant lesions;

2. Among doctors in dentistry, without doubt there is desperate need for additional education and update of old understandings about the premalignant lesions and oral cancer, as well as for new methods for early diagnosis;

3. Just like worldwide, as well as in the Republic of Macedonia there is need for decreasing the level of screening and early diagnosis of oral cancer from the tertiary health care facilities (Clinics for Maxillofacial Surgery) to the primary and secondary health care facilities (general dental offices and dental specialist offices);

4. It is necessary to make efforts, screening programs for oral cancer to enter the annual national program for screening of malignant diseases, thus adequate financial funds would be separated for its implementation. Therefore, preventive examinations for early detection of oral cancer would enter as a mandatory package in each dental office;

5. There is an obvious need for examining and publishing scientific papers about the large number of methods and tests for screening and early diagnosis of oral premalignant and malignant lesions in order to determine their sensitivity and specificity;

6. To actualize, advertise and scientifically support already proven methods and tests for screening, so the information about them would become available to a broad network of dentists in one state, and also to organize training courses for using these methods and tests;

7. Last but not least, we must raise the level of education and awareness among patients about this disease. For this purpose, it would be useful to organize forums in which citizens will learn about the clinical presentation, risk and the method for detection of this disease.

\section{Acknowledgements}

Publication fee for this review article was covered by Ss Cyril and Methodius University Skopje PhD School. 


\section{References}

1. Miloro M, Ghali GE, Larsen EP, Waite DP. Maxillofacial pathology. Peterson's principles of oral and maxillofacial surgery. Second edition. BC Decker Inc; Hamilton: London, 2004:1461(563-723).

2. Neville WB, Day AT. Oral cancer and precancerous lesions. CA Cancer J Clin. 2002; 52:195-215.

3. Messadi VD, Wilder-Smith P, Wolinsky L. improving oral cancer survival: The role of dental providers. J Calif Dent Assoc. 2009;37(11):789-798.

4. Huff K, Stark CP, Solomon WL. Sensitivity of direct tissue fluorescence visualization in screening for oral premalignant lesions in general practice. General Dentistry. Oral Diagnosis. 2008;34-38.

5. Lingen WM, Kalmar RJ, Karrison T, Speight MP. Critical Evaluation of Diagnostic Aids for the Detection of Oral Cancer. Oral Oncol. 2008;44(1):10-22.

6. Jemal A, Siegel R, et al. Cancer statistics, 2007. CA Cancer J Clin. 2007;57(1):43-66.

7. Jemal A, Siegel R, et al. Cancer statistics, 2008. CA Cancer J Clin. 2008;58(2):71-96.

8. Gillenwater A, Papadimitrakopoulou V, Richards-Kortum R. Oral premalignancy: New methods of detection and trearment. Curr Oncol Rep. 2006; 8(2): 146-154.

9. Mashberg A, Samit AM. Early detection, diagnosis, and management of oral and oropharyngeal cancer. CA Cancer J Clin. 1989; 39:67-88.

10. Silverman S, Gorsky M. Epidemiologic and demographic update in oral cancer: California and national data, 1973-1985. J Am Dent Assoc. 1990;120:495-9.

11. Alfano MC, Horowitz AM. Professional and community efforts to prevent morbidity and mortality from oral cancer. $\mathrm{J}$ Am Dent Assoc. 2001;132 (Suppl); 24S-29S.

12. Wong DT. Toward a simple, saliva based test for the detection of oral cancer. Expert Rev Mol Diagn. 2006; 6(3): 267-72.

13. Government of the Republic of Macedonia. Program for early detection of malignant diseases in Republic of Macedonia for 2012. Official gazette of RM No 38/91, 46/93, 55/95, 10/ 04, 84/05, 111/05, 65/06, 05/07, 77/08, 67/09, 88/10, 44/11 \& 53/11. 2012, January.

14. UK National Screening Committee. Criteria for appraising the viability, effectiveness and appropriateness of a Screening programme. http://www.nsc.nhs.uk/uk_nsc/uk_nsc_ind.htm

15. Wilson JM, Jungner YG. [Principles and practice of mass screening for disease]. Bol Oficina Sanit Panam. 1968;65(4):281-393. Spanish.
16. Horowitz AM. Perform a death-defying act: the 90-second oral cancer examination. J Am Dent Assoc. 2001;32(supplement): 36S-40S.

17. American Cancer Society, California Division and Public Health Institute, California Cancer Registry. California Cancer Facts and Figures 2007. American Cancer Society, California Division; Oakland; Sep, 2006.

18. Kramer IR, Lucas RB, Pindborg JJ et al. WHO Collaborating center for oral precancerous lesions. Definition of leukoplakia and related lesions. An aid to studies on oral precancer. Oral Surg Olar Med Oral Pathol. 1978; 46:518-539.

19. Banoczy J. Follow-up studies in oral leukoplakia. J Maxillofac Surg. 1977;5:69-75.

20. Silverman S, Bhargava K, Mani J, et al. Malignant transformation and natural history of oral leukoplakia in 57,518 industrial workers of Gujarat, India. Cancer. 1976;38:1790-5.

21. Silverman S, Rosen RD. Observations on the clinical characteristics and natural history of leukoplakia. J Am Dent Assoc. 1968;76:772-6.

22. Shafer WG, Waldron CA. Erythroplakia of the oral cavity. Cancer. 1975; 36:1021-1028.

23. Canniff JP, Harvey W, Harris M. Oral submucous fibrosis: its pathogenesis and management. Br Dent J. 1986;160:42934.

24. Epstein JB, Siverman SJr, et al. Analysis of oral lesion biopsies identified and evaluated by visual examination, chemiluminescence and toluidine blue. Oral Oncology. 2008; 44:538-44.

25. Rosenberg D, Cretin S. Use of meta-analysis to evaluate tolonium chloride in oral cancer screening. Oral Surg Oral Med Oral Pathol. 1987; 67:621-627.

26. Zheng W, Olivo M, Soo KC. The use of digitized endoscopic imaging of $5-A L A$-induced PPIX fluorescence to detect and diagnose oral premalignant and malignant lesions in vivo. Int J Cancer. 2004;110:295-300.

27. Li Y, St. John MAR, Zhou X, et al. Salivary transcriptome diagnostics for oral cancer detection. Clin Cancer Res. 2004; 10:8442-8450.

28. Farah CS, McCullough MJ. A pilot case control study on the efficacy of the acetic acid wash and chemiluminescent illumination (ViziLIte) in the visualization of oral mucosal white lesions. Oral Oncol. 2007; 43(8):820-4.

29. Kulapaditharom B, Boonkitticharoen V. Laser-induced fluorescence imaging in localization of head and neck cancers. Ann Otol Rhinol Laryngol. 1998;107:241-246.

30. Onizawa K, Saginoya H, Furuya Y, et al. Usefulness of fluorescence photography for diagnoses of oral cancer. Int J Oral Maxillofac Surg. 1999; 28:206-210. 
31. Hsu ER, Anslyn EV, Dharmawardhane S, et al. A far-red fluorescent contrast agent to image epidermal growth factor receptor expression. Photochem Photobiol. 2004;79:272-279.

32. Sokolov K, Follen M, Aaron J, et al. Real-time vital optical imaging of precancer using anti-epidermal growth factor receptor antibodies conjugated to gold nanoparticles. Cancer Res. 2003;63:1999-2004.
33. Soukos NS, Hamblin MR, Keel S, et al. Epidermal growth factor receptor-targeted immunophotodiagnosis and photoimmunotherapy of oral precancer in vivo. Cancer Res. 2001;61:4490-4496.

34. Wachsmann-Hogiu S, Weeks T, Huser T. Chemical analysis in vivo and in vitro by Raman spectroscopy - from single cells to humans. Curr Opin Biotechnol. 2009;20(1):63-73. 